Revista Brasileira de Tecnologias Sociais | ARTigos | DOI: 10.14210/RBTS.v5N1.P22-32

\title{
A SAÚDE E A SEGURANÇA DO TRABALHADOR SOB COMPETÊNCIA DE NORMAS REGULAMENTADORAS FRÁGEIS
}

\section{THE HEALTH AND SAFETY OF WORKERS UNDER THE COMPETENCE OF WEAK REGULATORY STANDARDS}

\author{
Larissa Maas ${ }^{1}$ \\ Luciane Peter Grillo² \\ Juliana Vieira de Araújo Sandri ${ }^{3}$
}

RESUMO: Os trabalhadores no Brasil têm seus direitos resguardados por meio das Normas Regulamentadoras do Ministério do Trabalho e Emprego. Este artigo analisa as fragilidades de duas normas referente à saúde do trabalhador e aos riscos ambientais, por meio de uma revisão documental a partir das Normas Regulamentadoras $n^{\circ} 7$ (PCMSO) e $n^{\circ} 9$ (PPRA), apontando-se como fragilidades os pontos que possam gerar interpretações equivocadas. As principais fragilidades encontradas foram: falta de esclarecimento nas ações de promoção da saúde; definição não explícita de responsáveis pela elaboração de documentos; informações imprecisas em relação aos documentos que devem ser realizados; falta de indicação de participação do trabalhador para elaboração dos documentos; e desatualização de aspectos importantes. Este estudo pode contribuir para o aprimoramento ou construção de uma nova legislação para a área de segurança e saúde do trabalhador.

PALAVRAS-CHAVE: Saúde ocupacional; Segurança do Trabalho; Legislação Trabalhista.

ABSTRACT: The rights of workers in Brazil are protected through the Regulatory Norms of the Ministry of Labor and Employment. This article analyzes the weaknesses of two norms relating to workers' health and environmental risks, through a documentary review based on Regulatory Norms no. 7 (PCMSO) and no. 9 (PPRA), pointing out, as weaknesses, some points that can generate misinterpretations. The main weaknesses were: a lack of clarification in health promotion actions; non-explicit definition of document makers; inaccurate information regarding the documents to be produced, lack of indication of the employee's participation in the preparation of the documents, and the outdatedness of some important aspects. This study may contribute to the improvement or construction of new legislation for the area of safety and health of workers.

Licença CC BY:

Artigo distribuído sob os termos Creative

Commons, permite uso e distribuição irrestrita em qualquer meio desde que $o$ autor credite a fonte original.
KEY WORDS: Occupational Health; Occupational Safety; Labor Legislation.

1 Engenheira Química. Mestre em Saúde e Gestão do Trabalho pela Universidade do Vale do Itajaí UNIVALI. Professora do Instituto Federal Catarinense - IFC, Campus Rio do Sul.

2 Nutricionista. Doutora em Pediatria e Ciências Aplicadas à Pediatria e Mestre em Nutrição pela Universidade Federal de São Paulo - UNIFESP. Professora titular da UNIVALI. E-mail: grillo@ univali.br.

3 Enfermeira. Doutora e Mestre em Enfermagem pela Universidade Federal de Santa Catarina - UFSC. Professora titular da UNIVALI. E-mail: jsandri@univali.br. 


\section{INTRODUÇÃO}

A saúde do trabalhador é um campo que está em constante evolução, estabelecendo a relação entre o processo saúde e trabalho, com características multidisciplinar, envolvendo profissionais de engenharia, medicina, psicologia, fisioterapia, direito, entre outros (MENDES; COSTA DIAS, 1991). O direito à saúde dos trabalhadores é assegurado por meio da legislação, que pode contribuir de forma significativa para a implantação de melhorias nessa área.

No Brasil, a legislação que garante o direito dos trabalhadores é a Consolidação das Leis do Trabalho (CLT), que especifica as regras da relação de contrato entre empresa e empregado. Diante da necessidade de esclarecer aspectos da CLT que precisavam ser explorados, em 1978 foram criadas as Normas Regulamentadoras (NR) do Ministério do Trabalho e Emprego (MTE) que estabelecem as condições mínimas para os ambientes de trabalho, a fim de promover a segurança e a saúde dos trabalhadores.

Assim, as NR são de execução obrigatória por parte das empresas privadas, podendo gerar falta grave passível de multa o não cumprimento do disposto na legislação. Atualmente, existem $36 \mathrm{NR}$ em vigor, cada uma com um tema distinto e atendendo segmento específico. Dentre as normas mais divulgadas estão a 7 - Programa de Controle Médico e Saúde Ocupacional (PCMSO), e a 9 - Programa de Prevenção de Riscos Ambientais (PPRA), que todas as empresas devem implantar (BRASIL, 1978).

A responsabilidade no cumprimento da legislação está atribuída ao governo, aos empregadores e aos trabalhadores. A responsabilidade solidária é um conceito largamente difundido na segurança do trabalho, com origem na CLT, que cita que a empresa deve "cumprir e fazer cumprir" as normas de segurança do trabalho (ARAÚJO-JÚNIOR, 2014)mas antes tenham os membros igual cuidado uns dos outros. De maneira que, se um membro padece, todos os membros padecem com ele; e, se um membro \u00e9 honrado, todos os membros se regozijam com ele. Ora, $\backslash \backslash$ uoof3s sois o corpo de Cristo, e seus membros em particular. (1 Cor \uooedntios 12, v. 25-27. Como exemplo, é possível perceber claramente a aplicação deste conceito na NR 7, quando cita a obrigatoriedade de a empresa contratante de serviços terceirizados informar os riscos existentes no local de trabalho, e consequentemente, auxiliar na elaboração e na implementação do PCMSO da empresa terceirizada.

Além disso, as NR encontram sua principal função na garantia do trabalho digno sem causar danos ou sofrimento ao trabalhador, portanto deve assegurar a subsistência do indivíduo, e ao mesmo tempo deve gerar realização profissional (SOUTO, 2011). Estudos apontam irregularidades e inconsistências em documentos ligados à área de segurança e de saúde do trabalhador (MIRANDA; DIAS, 2004; GONÇALVES; IGUTI, 2006; SILVA; SANTOS, 2014) e isso pode contribuir para que o ambiente de trabalho esteja inadequado, favorecendo o desenvolvimento de doenças.

As NR do MTE descrevem e identificam as condições do ambiente de trabalho para assegurar, no mínimo, a manutenção da saúde do trabalhador. Apesar de serem de observância obrigatória, há situações em que organizações não se enquadram nas exigências do disposto nas normas, mas ainda assim as NR podem servir como referência para o desenvolvimento das atividades em segurança do trabalho nestas instituições.

As discussões no campo saúde do trabalhador vêm crescendo nos últimos anos, mas ainda apresentam aberturas e espaços para ampliar os argumentos para a construção de políticas públicas 
no sentido das garantias dos direitos do trabalhador. Bezerra \& Neves (2010) apresentam um estudo que realizaram com artigos sobre a saúde do trabalhador, no qual apontam como resultado 28 artigos publicados no período de 2001 a 2008, com tema principal de discussão sobre políticas públicas e normas nessa área.

Além disso, as NR também apresentam aspectos pouco discutidos que podem levar a interpretações errôneas, e o descumprimento da lei pode conduzir a um número elevado de trabalhadores doentes e lesionados (SARDA et al., 2009; SALIBA, 2011; MENEGAT; CHASIN, 2013). Portanto, a discussão e a análise dos textos contribuem para esclarecimentos e alterações, facilitando a aplicação da legislação.

Este artigo propõe uma análise sobre o texto das NR 7 (PCMSO) e 9 (PPRA) que versam sobre programas de segurança e saúde do trabalhador. Com isso, é possível iniciar um processo de revisão destas ou construção de outras legislações que favoreçam a promoção da saúde do trabalhador.

\section{METODOLOGIA}

Estudo do tipo qualitativo, de caráter exploratório, sendo realizada pesquisa documental devido à natureza da fonte e porque os documentos ainda não receberam tratamento analítico (GIL, 2008), que neste caso são as NR 7 e 9, consultadas no site do MTE. A análise de documentos recebeu caráter parcial, apresentando-se aspectos importantes e pertinentes ao tema, buscandose elementos que possam contribuir para a discussão do problema, como melhorar a eficiência de implantação das NR 7 e 9 nas empresas e com isso contribuir para a saúde do trabalhador.

O objetivo, então, foi o de identificar as fragilidades que essas NR apresentam, gerando programas ineficientes, contribuindo ainda mais para o comprometimento da saúde do trabalhador.

A pesquisa foi dividida em duas etapas: análise do texto e identificação das fragilidades. Após leitura atenta do texto das NR, a análise documental foi realizada separando-se a norma pelos itens e identificando-se possíveis pontos de informações equivocadas ou que possam gerar interpretações distorcidas.

As fragilidades foram identificadas quando um item da NR não deixa evidente em seu texto o que pretende. Os itens selecionados para fragilidades na NR 7 foram: promoção da saúde, por ser um documento que deveria contribuir para a melhoria das condições de saúde do trabalhador; responsabilidades, tendo em vista que é um documento exigido por força de lei; e documentação, quando o texto não esclarece ou gera dúvidas de como elaborar os documentos necessários. E, para a NR 9, os itens foram: participação do trabalhador, levando-se em conta que é o maior atingido pela ação; riscos ambientais, que devem ser identificados nos ambientes laborais; e responsabilidades, igualmente como na análise da NR 7.

Este estudo limita-se a apontar aspectos das NR 7 e 9 que ainda foram pouco discutidos ou que merecem maior discussão para a compreensão apropriada do texto e, posteriormente, uma revisão para a elaboração de um novo texto da norma.

\section{RESULTADOS E DISCUSSÃO}

A legislação deve apresentar texto claro e conciso para não gerar interpretações diversas, que podem acarretar a falta de cumprimento dos itens por ela preconizados. A atuação dos 
profissionais desta área se embasa nas normas (PEETERS et al., 2003) e vale ressaltar que a falta de preparo técnico também gera dificuldades de implantação de ações no campo saúde do trabalhador (LOURENÇO; LACAZ, 2013).

Alguns termos utilizados no tex to da legislação deixam margem para várias interpretações, como observado por Morais (2010), que quando a norma menciona que a empresa "pode" realizar tal atividade para atingir o objetivo, não necessariamente se refere ao que a empresa "deve" obrigatoriamente desenvolver.

Diversos estudos já foram realizados verificando-se a baixa qualidade técnica dos documentos de segurança e saúde do trabalho, como a falta de cumprimento das exigências legais descritas nas NR e as inconsistências nos documentos (MIRANDA; DIAS, 2004; LIMA et al., 2007; CHAVES et al., 2009) que em conjunto com outros fatores colaboram para a negligência da saúde do trabalhador. Apesar dos estudos citados terem sido realizados entre 2000 e 2009, podese adotar os resultados como atuais, sendo que os textos básicos da NR 7 e 9 são do ano de 1994, com poucas revisões posteriores.

\section{Norma Regulamentadora 7 - Programas de Controle Médico de Saúde Ocupacional} (PCMSO)

A NR 7 descreve o documento, intitulado PCMSO, que deve contemplar os exames médicos recomendados para cada função existente na empresa, as ações de saúde a serem implantadas pela empresa e outras recomendações importantes para a manutenção da saúde do trabalhador. O principal objetivo do PCMSO deve ser de prevenção, rastreamento e diagnóstico precoce de doenças do trabalho, utilizando-se preferencialmente do instrumental clínico-epidemiológico no momento de análise das relações saúde-trabalho. O responsável pela elaboração do PCMSO é o médico do trabalho, que deve ser um médico com especialização em medicina do trabalho (BRASIL, 2013).

O texto da NR inicia indicando que todas as empresas que admitam trabalhadores como empregados devem elaborar o PCMSO (item 7.1.1), ou seja, todas as empresas de iniciativa privada. Todavia, as empresas que não são regidas pela Consolidação das Leis do Trabalho (CLT) não estão obrigadas a cumprir tal norma (BRASIL, 2013), evidenciando o abandono, por parte da legislação, da segurança e da saúde de determinados grupos de trabalhadores (RAMMINGER; NARDI, 2014).

Os exames mínimos obrigatórios são: admissional, periódico, retorno ao trabalho, mudança de função e demissional (item 7.4.1). A partir dos exames periódicos, deve ser elaborado um relatório anual com os resultados para monitoramento e implantação de ações. Caso ocorra algum exame alterado, um Comunicado de Acidente de Trabalho (CAT) deve ser emitido (item 7.4.8), para evidenciar o risco do ambiente de trabalho (BRASIL, 2013). No entanto, quando um exame médico apresenta resultado alterado, este trabalhador já está contaminado ou doente, o que confirma que as medidas de controle do risco não estão sendo eficientes.

Além disso, o exame médico é importante ferramenta para avaliação das condições físicas e mentais do indivíduo, e indica se o trabalhador está apto a desenvolver determinada tarefa. Historicamente, o exame médico foi amplamente utilizado em favor da maior produtividade que o indivíduo pudesse ter, como descrito por Berlinguer (1988), que no ano de 1927, na Itália, o médico da fábrica deveria examinar os trabalhadores a serem admitidos, a fim de identificar se tinham resistência suficiente para os agentes nocivos que iriam estar expostos. Souto (2011) observa que 
a atuação dos médicos do trabalho limita-se à realização do mínimo possível nas ações ligadas à saúde do trabalhador, e defende que essa atuação deveria ser estendida. Ainda para o mesmo autor, o sistema mercantilista gerado no campo de segurança e saúde do trabalhador, valorizando a quantidade e não a qualidade nos serviços prestados, é o fator responsável pelo modo de agir dos profissionais da área, o que pode ainda mais limitar as ações em favor da saúde do trabalhador.

Atualmente, novos modelos têm sido propostos para auxiliar os médicos do trabalho para uma maior efetividade, como a utilização de instrumentos específicos no exame médico para atividades de trabalho em altura, definidas como atividades de alto risco (HAYASHIDE; BUSCHINELLI, 2017).

\section{Promoção DA SAÚde}

Para Bellusci (2013), a prevenção de doenças, as perdas da capacidade funcional e a qualidade do envelhecimento podem ser melhoradas com a promoção da saúde do trabalhador. Assim, há a necessidade de difundir o conceito de promoção à saúde para os profissionais da área de segurança do trabalho, e com isso ampliar as ações voltadas à qualidade de vida destes trabalhadores.

Um dos objetivos da NR 7 é a promoção e a preservação da saúde dos trabalhadores (item 7.1.1). Embora o termo promoção da saúde seja citado, torna-se claro somente a preservação da saúde, já que as ações de promoção não estão explícitas na norma, pois nos itens obrigatórios do PCMSO nada sobre o tema é mencionado. Além dos benefícios na qualidade de vida para os trabalhadores, os programas de promoção à saúde apresentam melhoria de produtividade e redução de absenteísmo, rotatividade e doenças (SILVA, 2014; FERRAZ et al., 2017). A explicação sobre ações e percursos para a promoção da saúde dos trabalhadores dentro das empresas possibilitaria maior compreensão pelos profissionais para aplicação da norma.

\section{RESPONSABILIDADES}

Para contribuir com a promoção à saúde dos trabalhadores, outros profissionais da área da saúde estão aptos a desenvolver programas com esta finalidade. Além do médico do trabalho, o enfermeiro do trabalho, que tem sua formação baseada em gerenciamento dos riscos, da promoção e da reabilitação da saúde dos trabalhadores (SILVA et al., 2016). Segundo Lino e colaboradores (2012), as atribuições dessa categoria profissional não estão delimitadas, mas desde 1978 as definições da profissão de enfermeiro do trabalho já haviam sido criadas. Como forma de esclarecimento, a norma poderia citar o enfermeiro do trabalho como o profissional que apresenta capacidade para realizar a gestão das informações obtidas no PCMSO, inclusive monitorando os resultados dos exames médicos e as informações do relatório anual.

Salienta-se ainda que todas as empresas devem ter equipamentos de primeiros socorros, que devem permanecer em local adequado, sob os cuidados de um trabalhador treinado para manusear tais equipamentos (item 7.5.1) (BRASIL, 2013). As informações detalhadas sobre carga horária, qual profissional deve ministrar o treinamento, validade e reciclagem, não estão definidas, permitindo diversos profissionais da área da saúde a responsabilidade sobre estes elementos do curso, dentre eles o enfermeiro ou profissional de educação física (OLIVEIRA et al., 2015; SALES et al., 2016). Essa lacuna nas informações pode conduzir as ações tanto para sobrecarga quanto para insuficiência na realização do treinamento. 


\section{DOCUMENTAÇÃO}

As empresas que não apresentam risco grave aos trabalhadores estão desobrigadas de indicar médico coordenador (item 7.3.1.1), conforme apresentado no Quadro 1, e ainda conforme a norma prevê, as empresas que não têm médico coordenador não precisam elaborar o relatório anual com os resultados dos exames médicos (item 7.4.6.4). Ou seja, empresas que não possuam risco grave em seu ambiente de trabalho ficam desobrigadas de elaborar o relatório anual do PCMSO.

Quadro 01: Empresas desobrigadas de indicar médico coordenador

\begin{tabular}{|l|l|}
\hline Número de funcionários & Grau de risco $\mathbf{1}$ e $\mathbf{2}$ \\
\hline Até 25 & Desobrigada \\
\hline De 25 a 50 & Negociável \\
\hline \multicolumn{2}{|l|}{} \\
\hline Número de funcionários & Grau de risco 3 e 4 \\
\hline Até 10 & Desobrigada \\
\hline De 10 a 20 & Negociável \\
\hline
\end{tabular}

Fonte: Elaborado pelas autoras.

O relatório anual é importante ferramenta para gerar mudanças na área de saúde do trabalhador, pois o médico identifica as alterações nos resultados de exames médicos e isso acarreta ações para melhorar os controles dos riscos no ambiente de trabalho, sob uma visão completa da empresa. Como exemplo, empresas que se enquadram nessa situação são: coleta de resíduos perigosos, construção de edifício, extração de carvão mineral e fabricação de cimento (BRASIL, 2013), que, apesar de apresentarem muitos riscos geradores de doenças em seus ambientes de trabalho, quando há poucos trabalhadores, estão isentas da elaboração do relatório anual e os benefícios que pode trazer para o controle dos riscos.

Outro aspecto que a legislação indica é que nas localidades que não há médico do trabalho, médico de outra especialidade poderá ser médico coordenador do PCMSO (item 7.3.1). Para não gerar uma falha na identificação dos riscos geradores de doenças ao trabalhador, caberia uma observação em relação à supervisão deste documento por um médico do trabalho, para a garantia da análise do PPRA e integração dos documentos de segurança.

\section{Norma Regulamentadora 9 - Programas de Prevenção de Riscos Ambientais}

O documento descrito nessa NR é o PPRA, deve ser elaborado por todas as empresas que admitam trabalhadores com relação de trabalho regida pela CLT (item 9.1.1) (BRASIL, 2017), ou seja, igualmente ao PCMSO, as empresas da iniciativa privada. Mais uma vez as organizações que não são regidas pela CLT ficam desobrigadas do cumprimento desta legislação (RAMMINGER; NARDI, 2014).

Segundo Saliba (2011), o PPRA é um documento de extrema importância para a gestão das condições do ambiente de trabalho com objetivo de prevenir doenças, mas deve ser bem elaborado pelo profissional da segurança ou medicina do trabalho. O autor ainda salienta que a 
interpretação equivocada é um dos principais fatores contribuintes para a visão distorcida de que o programa é apenas um documento a ser apresentado no momento de fiscalização do auditor do MTE. Portanto, a legislação deve ser tão clara quanto possível para contribuir para a implantação adequada dos programas de segurança.

Além disso, o PPRA é um documento que serve como referência para o desenvolvimento de ações na área de segurança do trabalho. Esse documento, como seu próprio nome sugere, está ligado ao planejamento do método de trabalho para a prevenção de riscos que podem gerar algum dano aos indivíduos, equipamentos, ou local de trabalho.

\section{ParticipaÇÃo do trabalHador}

A norma cita a importância da participação do trabalhador nas etapas de planejamento e execução do documento PPRA (item 9.6.2) (BRASIL, 2017). Apesar disso, a participação do trabalhador é restrita, as ações dos trabalhadores nas avaliações não são levadas em consideração, o que pode resultar na falta de conscientização dos riscos do ambiente pelos trabalhadores. No estudo realizado por Miranda \& Dias (2004), identificou-se que não há contribuição do trabalhador para a elaboração dos documentos de segurança do trabalho. Situação que poderia ser revista com um texto explícito sobre as formas de participação do trabalhador.

\section{RISCOS AMBIENTAIS}

A NR 9 considera riscos ambientais os agentes físicos, químicos e biológicos, que podem causar danos à saúde do trabalhador (item 9.1.5). Os agentes físicos são: ruído, vibrações, radiações, pressões anormais e temperaturas extremas, infrassom e ultrassom. Os agentes químicos são poeiras, fumos, névoas, neblinas, gases e vapores. Os agentes biológicos são bactérias, fungos, bacilos, parasitas, protozoários, vírus, entre outros (BRASIL, 2017).

Apesar disso, os riscos do Mapa de Riscos devem ser levados em consideração para o planejamento e a execução do PPRA (item 9.6.2) (BRASIL, 2017). A legislação de segurança, por meio da Portaria $n^{\circ}$ 25/1994, que organiza o funcionamento do Mapa de Risco, aponta, além dos citados na NR 9, os riscos ergonômicos e de acidentes (BRASIL, 1994). Os Mapas de Risco são documentos elaborados por integrantes da Comissão Interna de Prevenção de Acidentes (CIPA), identificando-se os riscos físicos, químicos, biológicos, ergonômicos e mecânicos, quando encontrados no ambiente de trabalho (BRASIL, 1994). Ou seja, é possível indicar também os riscos ergonômicos e mecânicos no PPRA, apesar de não estarem citados no corpo do texto da NR 9. Para evitar interpretações equivocadas, as definições de risco deveriam ser atualizadas para o entendimento claro por profissionais da área, levando a uma avaliação completa dos riscos existentes no ambiente.

Paralelamente, a NR 17 - "Ergonomia” versa sobre riscos ergonômicos, a NR 20 "Segurança e Saúde no trabalho com Inflamáveis e combustíveis” cita risco de acidente, bem como a NR 12 - "Segurança no trabalho com máquinas e equipamentos" cita risco mecânico, também indicando a inclusão desse risco no PPRA.

Além disso, a Organização Mundial da Saúde (OMS) aponta os agentes ergonômicos e psicossociais como geradores de doenças aos trabalhadores (SULZBACHER; FONTANA, 2013). Para Bellusci (2013), as condições de risco que o ambiente pode oferecer são: ambiente 
físico, presença de substâncias tóxicas no ambiente, presença de agentes biológicos, características antropométricas do ambiente, e características de organização do trabalho. Já para Souto (2011), as causas que podem gerar doenças ocupacionais são mecânicas, físicas, químicas, biológicas, psicogênicas (estímulos fortes e prolongados) e genéticas. Rouquayrol \& Gurgel (2013), bem como o Ministério da Saúde no manual de Doenças Relacionadas ao Trabalho (MINISTÉRIO DA SAÚDE DO BRASIL, 2001), reconhecem os riscos ergonômicos e de acidentes como possíveis geradores de doenças ao trabalhador. Então, o texto da NR 9 carece atualização sobre novos riscos que são amplamente reconhecidos (LEITÃO et al., 2009; CHIODI et al., 2010; RUIZ; ARAUJO, 2012).

\section{Responsabilidades}

Quando se discute o tema segurança e saúde do trabalhador, a responsabilidade pelos documentos, programas e ações que devem ser realizados precisa estar bem definida, pois qualquer omissão ou ato falho pode prejudicar consideravelmente a vida dos trabalhadores.

A elaboração de um documento de segurança do trabalho é de extrema importância e deve ser realizada por um profissional especializado que tenha o entendimento de suas responsabilidades quando realiza um trabalho dessa amplitude. Os profissionais da área de segurança do trabalho apresentam conhecimento específico para atender aos requisitos solicitados na legislação e podem assumir essa responsabilidade (BRASIL, 1978). Quando, no texto da NR 9, é citado que a empresa pode indicar profissional que julgar apto a elaborar o PPRA para fazê-lo (item 9.3.1.1), o documento elaborado pode não cumprir seu principal objetivo, que é, no mínimo, a preservação da saúde do trabalhador. Novamente a interpretação fica comprometida devido à imprecisão das informações (SOUTO, 2011).

Em síntese, é possível observar no Quadro 2 os principais aspectos apontados que podem ser esclarecidos nos textos das NR para sua melhor aplicação. Além disso, todas as empresas deveriam ser obrigadas a cumprir esta legislação, mesmo aquels que não são regidas pela CLT, isso aumentaria o núumero de trabalhadores amparados por uma legislação específica.

Quadro 02: Resumo das fragilidades encontradas

\begin{tabular}{|l|l|}
\hline \multicolumn{2}{|c|}{ NR 7 - PCMSO } \\
\hline Item & \multicolumn{1}{|c|}{} \\
\hline Promoção à saúde & $\begin{array}{l}\text { As ações de promoção à saúde poderiam estar exemplificadas e mais } \\
\text { esclarecidas no texto. }\end{array}$ \\
\hline Responsabilidades & $\begin{array}{l}\text { O enfermeiro do trabalho poderia ser sugerido como gestor de ações } \\
\text { no PCMSO, e organizador do treinamento de primeiros socorros. }\end{array}$ \\
\hline Documentação & $\begin{array}{l}\text { Todas as empresas deveriam ser obrigadas a realizar o relatório } \\
\text { anual, independentemente do número de funcionários, como forma de } \\
\text { monitoramento e controle das condições de saúde dos trabalhadores. } \\
\text { Além disso, o responsável pelo PCMSO sempre deveria ser médico do } \\
\text { trabalho, mesmo que em conjunto com médico de outra especialidade. }\end{array}$ \\
\hline \multicolumn{2}{|c|}{ NR 9 - PPRA } \\
\hline Item & Sugestões \\
\hline Participação do trabalhador & $\begin{array}{l}\text { A percepção do risco pelo trabalhador deveria ser levada em } \\
\text { consideração no momento de avaliação de riscos. }\end{array}$ \\
\hline Riscos ambientais & $\begin{array}{l}\text { É necessária uma revisão para a inclusão de novas formas de riscos } \\
\text { que podem gerar doenças ou acidentes aos trabalhadores. }\end{array}$ \\
\hline
\end{tabular}




\begin{tabular}{|l|l|}
\hline Responsabilidades & $\begin{array}{l}\text { Somente os profissionais da área é que possuem conhecimento técnico } \\
\text { para elaborar um documento tão importante, portanto, deveria estar } \\
\text { indicado no texto da NR os profissionais aptos a realizar tal tarefa. }\end{array}$ \\
\hline
\end{tabular}

Fonte: Elaborado pelos autores.

\section{CONCLUSÃO}

Neste estudo, a partir de uma revisão documental das NR 7-PCMSO e 9-PPRA, identificaramse fragilidades que as normas podem apresentar e buscaram-se discutir os pontos a serem melhorados na legislação. A escassez de literatura em legislação em segurança e saúde do trabalhador indica a discussão precoce e a carência de atualização da área. Essa pesquisa limitou-se a apontar aspectos das normas pouco discutidos, e com isso estimular a revisão do texto das normas citadas.

Embora a legislação deva ser entendida como referência para o planejamento das ações em segurança e saúde do trabalhador, se não estiver clara, dificulta a realização das ações em segurança. Apesar de o texto descrever os requisitos mínimos a serem cumpridos, um conteúdo claro facilita o entendimento de todos os profissionais da área e dos trabalhadores envolvidos.

A reformulação das NR com texto mais claro e de fácil entendimento pode contribuir para a ampliação e divulgação das informações do cuidado com a saúde do trabalhador. As NR são de fácil acesso, uma vez que é possível acessá-las pela internet, apenas precisam ser de fácil entendimento e conter informações mais claras para que a compreensão gere ações que irão contribuir para a manutenção da saúde dos trabalhadores.

\section{REFERÊNCIAS}

ARAÚJO-JÚNIOR, F. A terceirização e o descompasso com a higidez, saúde e segurança no meio ambiente laboral - responsabilidade solidária do tomador do serviço a partir das normas de saúde e segurança no trabalho. Revista Tribunal Regional do Trabalho, v.58, n.89, p.67-81, 2014.

BELLUSCI, S. M. Doenças profissionais ou do trabalho. 12.ed. São Paulo: Senac São Paulo, 2013.

BERLINGUER, G. A doença. São Paulo: HUCITEC, 1988.

BEZERRA, M. L. S.; NEVES, E. B. Perfil da Produção Científica em Saúde do Trabalhador. Saúde e Sociedade, São Paulo, v.19, n.2, p.384-394, 2010.

BRASIL. Portaria 3.2 14, de 8 de junho de 1978. Brasília: Normas Regulamentadoras do Ministério do Trabalho, 1978.

BRASIL. Portaria n 25, de 29 de dezembro de 1994 - Anexo IV Mapa de riscos. Brasília: Normas Regulamentadoras do Ministério do Trabalho, 1994.

BRASIL. Norma Regulamentadora 7 - Programa de Controle Médico de Saúde Ocupacional (PCMSO). Brasília: Normas Regulamentadoras do Ministério do Trabalho, 2013.

BRASIL. Norma Regulamentadora 9 - Programa de Prevenção de Riscos Ambientais (PPRA). Brasília: Normas Regulamentadoras do Ministério do Trabalho, 2017.

CHAVES, S. C. L.; SANTANA, V. S.; LEÃO, I. C. M. DE; SANTANA, J. N. DE; LACERDA, L. M. A. DE A. Determinantes da implantação de um programa de segurança e saúde no trabalho. Revista Panamericana de Salud Pública, v.25, n.3, p.204-212, 2009. 
CHIODI, M. B.; MARZIAle, M. H. PALUCCI; MONDADORI, R. M.; ROBAZZI, M. L. DO C. C. Acidentes registrados no Centro de Referência em Saúde do Trabalhador de Ribeirão Preto, São Paulo. Revista Gaúcha de Enfermagem, v.31, n.2, p.211-217, 2010.

FERRAZ, J. L.; NUNES, P. L.; GOULART JÚNIOR, E.; CAMARGO, M. L. Reflexões acerca da necessidade da promoção da saúde nos ambientes de trabalho. Revista OMNIA Saúde, v.13, n.1, p. 10$25,2017$.

GIL, A. C. Métodos e Técnicas de Pesquisa Social. 6.ed. São Paulo: Atlas, 2008.

GONÇALVES, C. G. DE O.; IGUTI, A. M. Análise de programas de preservação da audição em quatro indústrias metalúrgicas de Piracicaba, São Paulo, Brasil. Cadernos de Saúde Pública, v.22, n.3, p.609$618,2006$.

HAYASHIDE, J. M.; BUSCHINELLI, J. T. P. Critérios de decisão para a definição de exames médicos ocupacionais em atividades críticas: proposição de modelo e exemplos de aplicação no trabalho em altura. Revista Brasileira de Saúde Ocupacional, v.42, p.1-16, 2017.

LEITÃO, I. M. T. A.; FERNANDES, A. L.; RAMOS, I. C. Saúde ocupacional: analisando os riscos relacionados à equipe de enfermagem numa unidade de terapia intensiva. Ciência, Cuidado e Saúde, v.7, n.4, p.476-484, 2009.

LiMA, M. M. R.; DANTAS, R. A.; PAGLiUCA, L. M. F.; ALMEIDA, P. C. DE. Programa de Controle Médico de Saúde Ocupacional em Companhias Elétricas Brasileiras. Revista RENE, v.8, n.3, p.61-68, 2007.

LINO, M. M.; NORA, P. T.; LINO, M. M.; FURTADO, M. Enfermagem do Trabalho à Luz da Visão Interdisciplinar. Saúde \& Transformação Social, v.3, n.1, p. 85-91, 2012.

LOURENÇO, E. Â. DE S.; LACAZ, F. A. DE C. Os desafios para a implantação da política de Saúde do Trabalhador no SUS: o caso da região de Franca-SP. Revista Brasileira de Saúde Ocupacional, v.38, n.127, 2013.

MENDES, R.; COSTA DIAS, E. DA. Da medicina do trabalho à saúde do trabalhador. Revista de Saúde Pública, v.25, n.5, p. 341-349, 1991.

MENEGAT, F. D.; CHASIN, A. A. DA M. Estudo comparativo das Normas Regulamentadoras da relação entre trabalho, saúde e doença, em serviços de saúde. RevInter Revista Intertox de Toxicologia, Risco Ambiental e Sociedade, v.6, n.1, p.115-131, 2013.

MINISTÉRIO DA SAÚDE DO BRASIL. DOENÇAS RELACIONADAS AO TRABALHO - Manual de Procedimentos para os Serviços de Saúde. Brasília, 2001.

MIRANDA, C. R.; DIAS, C. R. PPRA/PCMSO: auditoria, inspeção do trabalho e controle social. Cadernos de Saúde Pública, v.20, n.1, p.224-232, 2004.

MORAIS, C. R. N. Perguntas e respostas comentadas em segurança e medicina do trabalho. São Caetano do Sul: Yendis, 2010.

OLIVEIRA, M. R. DE; LEONEL, A. R. A.; MONTEZELI, J. H.; et al. Conception of undergraduate nursing students on the practice of health education on first aid. Revista da Rede de Enfermagem do Nordeste, v.16, n.2, p.150-158, 2015.

PEETERS, S. E.; DUARTE, F. J. C. M.; GARRIGOU, A.; et al. Segurança do Trabalho: que trabalho é esse? XXIII Encontro Nacional de Engenharia de Produção, 2003. 
RAMMINGER, T.; NARDI, H. C. Saúde do trabalhador: um (não) olhar sobre o servidor público. Revista do Serviço Público, v.58, n.2, p.213-226, 2014.

ROUQUAYRoL, M. Z.; GURGEL, M. Epidemiologia e Saúde. 7.ed. Rio de Janeiro: MedBook, 2013.

RUIZ, V. S.; ARAUJO, A. L. L. DE. Saúde e segurança e a subjetividade no trabalho: os riscos. Revista Brasileira de Saúde Ocupacional, v.37, n.125, 2012.

SALES, J. S. DE; SILVA, G. H.; PEREIRA, J. P. B.; JUNIOR, M. F. D.; MEDEIROS, D. C. DE. Formação de professores e nível de conhecimento de professores de educação física escolar sobre os primeiros socorros na cidade do Natal/RN. Revista Humano Ser, v.1, n.1, p. 49-63, 2016.

SALIBA, T. M. Curso básico de segurança e higiene ocupacional. São Paulo: LTR, 2011.

SARDA, S. E.; RUIZ, R. C.; KIRTSCHIG, G. Tutela jurídica da saúde dos empregados de frigoríficos: considerações dos serviços públicos. Acta Fisiátrica, v.16, n.2, p.59-65, 2009.

SILVA, E. S. N.; SANTOS, T. F. V. DOS. Análise dos padrões técnicos de Programas de Controle Médico de Saúde Ocupacional e Atestados de Saúde Ocupacional por meio de auditoria interna. Revista Brasileira de Medicina do Trabalho, v.12, n.2, p.50-56, 2014.

SILVA, P. S. C. DA. Programas de promoção da saúde do trabalhador e suas influências: uma revisão de literatura. Revista Eletrônica Gestão \& Saúde, v.5, n.2, p.727-752, 2014.

SILVA, R. P.; VALENTE, G. S. C.; BARRETO, B. M. F.; CAMACHO, A. C. L. F.; CAMACHO, A. C. L. F. O gerenciamento de riscos ocupacionais e as interferências na saúde do trabalhador: revisão integrativa. Revista de Pesquisa: Cuidado é Fundamental Online, v.8, n.2, p.4168, 2016.

SOUTO, D. Saúde no trabalho: uma revolução em andamento. 2ed. Rio de Janeiro: Sesc Nacional, 2011.

SULZBACHER, E.; FONTANA, R. T. Concepções da equipe de enfermagem sobre a exposição a riscos físicos e químicos no ambiente hospitalar. Revista Brasileira de Enfermagem, v.66, n.1, p. 25-30, 2013. 